\title{
Calorie restriction for long-term remission of type 2 diabetes
}

\author{
Author: Roy Taylor ${ }^{\mathrm{A}}$
}

Starting with a hypothesis which postulated a simple explanation arising from the basic cause of type 2 diabetes, a series of studies has introduced a paradigm shift in our understanding of the condition. Gradual accumulation of fat in the liver and pancreas leads eventually to beta cell dedifferentiation and loss of specialised function. The consequent hyperglycaemia can be returned to normal by removing the excess fat from liver and pancreas. At present this can be achieved only by substantial weight loss, and a simple practical and efficacious method for this has been developed and applied in a series of studies. For those people who used to have type 2 diabetes, the state of post-diabetes can be long term provided that weight regain is avoided. The implications for personal health and for national health economics are considerable.

KEYWORDS: Type 2 diabetes, reversal of type 2 diabetes, liver fat, pancreas fat, first phase insulin response

\section{Established beliefs about type 2 diabetes}

Type 2 diabetes has long been regarded as a lifelong condition which is inexorably progressive. Despite modern treatments, longterm complications remain common and devastating. These clinical observations are backed up by detailed studies showing that $50 \%$ of people require insulin therapy after 10 years of type 2 diabetes. ${ }^{1}$ The underlying pathophysiology of type 2 diabetes seemed clear: insulin resistance together with a steadily progressive beta cell defect. The United Kingdom Prospective Diabetes Study (UKPDS) showed that beta cell function had declined to around $50 \%$ of normal by the time of diagnosis, ${ }^{2}$ and continued to decline irrespective of pharmacological treatments. ${ }^{3}$ Histological studies using insulin immunostaining to identify beta cells appeared to confirm this with a $50 \%$ decrease in beta cell number at diagnosis. ${ }^{4}$ This was presumed to reflect continued death or apoptosis of beta cells. To assess whole body insulin resistance, the insulin clamp became regarded as the gold standard method, even though this predominantly assesses muscle. Insulin resistance in muscle was established as the earliest detectable warning sign that type 2 diabetes may develop. ${ }^{5}$ The predominant focus of research has therefore been to identify better ways of improving muscle insulin

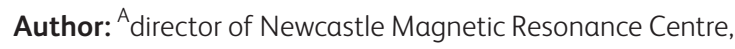
Newcastle University, Newcastle upon Tyne, UK resistance by drugs (eg thiazolidinediones). The apparent loss of beta cells appeared impervious to therapeutic intervention.

The consequence has been that people are told at diagnosis that they must get used to having a chronic disease, with major risk to eyesight, feet, heart and brain. But these entrenched beliefs were based only on data from groups of people who steadily gained weight (approximately $5 \mathrm{~kg}$ in the intensively treated group of UKPDS).

\section{The twin cycle hypothesis}

Research on liver insulin resistance in relation to liver fat content offered a potential breakthrough in understanding; the higher the liver fat, the greater the insulin resistance of the liver. ${ }^{6,7}$ Given that insulin action regulates hepatic glucose output, excess fat in the liver could explain fasting hyperglycaemia. But how about the beta cell defect which was always associated with development of type 2 diabetes? Surely a disease as common as type 2 diabetes must have a single cause, and not separate disease processes in different organs? What if excess fat also caused dysfunction of the beta cell? The twin cycle hypothesis applied Occam's razor (Fig 1). ${ }^{8}$ The hypothesis could easily be tested by a clinical study, and the COUNTERacting the Pancreatic inhibition Of INsulin secretion by Triglyceride (Counterpoint) study was launched. ${ }^{9}$

It was powered to detect a significant fall in fasting plasma glucose levels in a small group size $(n=11)$. To test the underlying mechanisms, new methodology was developed to measure fat within the substance of the pancreas using advanced magnetic resonance techniques. The results were clear-cut: individuals with very ordinary type 2 diabetes (up to 4 years duration) regained absolutely normal fasting glucose levels within 7 days of commencing a very low calorie diet. This was despite stopping metformin therapy on day one of the diet. Liver fat levels dropped by $30 \%$ and liver insulin sensitivity returned to normal in the first 7 days, explaining the change in fasting plasma glucose. However, the change in insulin secretion followed a different time course, gradually improving to normal over 8 weeks. The main data from Counterpoint are illustrated in Fig 2.

\section{Is remission of diabetes durable?}

It was clearly necessary to determine whether the reversal of underlying pathophysiology and resulting normoglycaemia would persist in the longer term. The basic requirement to achieve nondiabetic glucose control was weight loss of $15 \mathrm{~kg}$. Although this may seem unattainable, a pragmatic approach to permit success during everyday life had been developed in order to carry out the Counterpoint study. ${ }^{9}$ To answer the question of durability, it was 


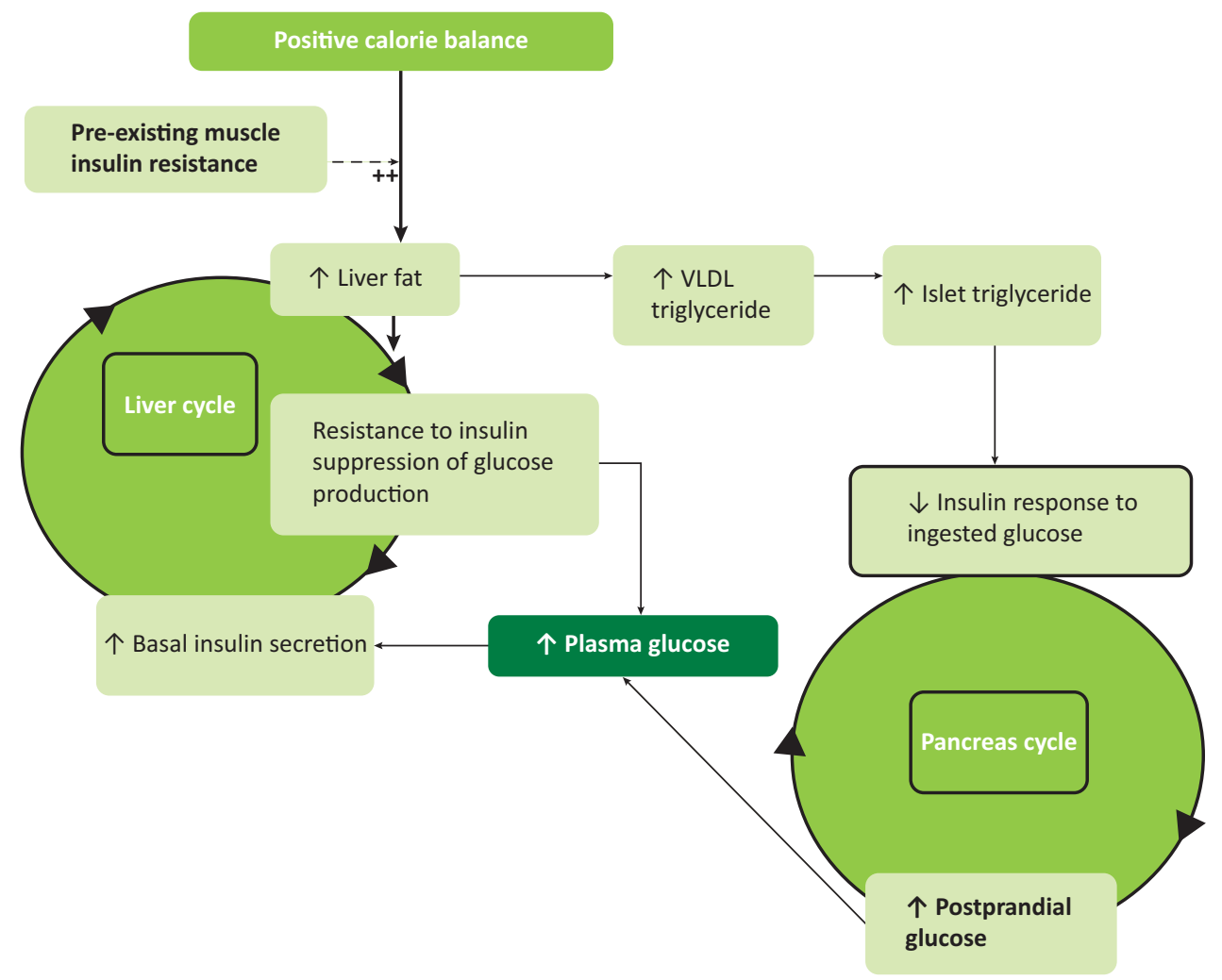

Fig 1. The twin cycle hypothesis: during long-term intake of more calories than are expended each day, excess carbohydrate must undergo de novo lipogenesis, which particularly promotes fat accumulation in the liver. As insulin stimulates de novo lipogenesis, people with insulin resistance (determined by family or lifestyle factors) will accumulate liver fat more readily than others due to the higher plasma insulin levels. The increased liver fat will cause relative resistance to insulin-suppression of hepatic glucose production. Over many years a modest increase in fasting plasma glucose level will cause increased basal insulin secretion rates as a homeostatic response. The consequent hyperinsulinemia will increase further the conversion of excess calories into liver fat. A vicious cycle of hyperinsulinemia and inadequate suppression of hepatic glucose production becomes established. Fatty liver leads to increased export of very low density lipoprotein (VLDL) triacylglycerol which will increase fat delivery to all tissues including the islets. Excess fatty acid availability in the pancreatic islet impairs the acute insulin secretion in response to ingested food, and at a certain level of fatty acid exposure, dedifferentiation of the beta cell will occur with post-prandial hyperglycaemia. The hyperglycaemia will further increase insulin secretion rates, with secondary increase of hepatic lipogenesis, spinning the liver cycle faster and driving on the pancreas cycle. Eventually the fatty acid and glucose inhibitory effects on the islets reach a trigger level for dedifferentiation, leading to a relatively sudden onset of clinical diabetes. Figure adapted with permission from R Taylor. ${ }^{8}$

necessary to optimise the transition from the 8 week very low calorie diet to a way of eating which would maximise the chance of maintaining constant weight long term. In the COUNTERacting BetA cell failure by Long term Action to Normalize Calorie intakE (Counterbalance) study, a stepped food reintroduction guided the return to normal foodstuffs with intensive education. ${ }^{10}$ In this larger group of 30 people, similar weight loss was attained, and in those returning to non-diabetic glucose control (notably those with shorter duration of diabetes) there was zero weight regain by 6 months. The improvement in liver fat, pancreas fat and first phase insulin secretion remained constant and consequently nondiabetic levels of $\mathrm{HbA} 1 \mathrm{c}$ were maintained during this period.

The encouraging results from Counterbalance have been extended by the randomised Diabetes REmission Clinical Trial (DiRECT)..$^{11}$ This large study was conducted by primary care nurses, or dietitians if available, and the intervention consisted of a structured 8 hours training in how to achieve the necessary weight loss and prevent weight regain. At 12 months, $24 \%$ achieved weight loss of more than $15 \mathrm{~kg}$ in the intervention group. $46 \%$ of the intervention group had non-diabetic $\mathrm{HbA} 1 \mathrm{c}$ off all antihyperglycaemic agents. The underlying changes in intra-organ fat remained constant in those achieving remission (Fig 3). Remission primarily depended upon degree of weight loss, with achievement increasing steadily from (7\%) with only $0-5 \mathrm{~kg}$ weight loss to $86 \%$ who lost $15 \mathrm{~kg}$ or more. The accompanying improvement in vitality was much appreciated by the participants, as was the escape from the burden of tablets to swallow daily.

Substantial weight loss achieved by any means can bring about remission of type 2 diabetes, and this has been observed most comprehensively in the Swedish obesity study. ${ }^{12}$ The belief about glucose lowering effects are due to GLP-1 changes after surgery rather than simple release from fat-induced beta cell suppression have been shown to be incorrect. ${ }^{13,14}$

It is important to note in passing that all anti-hypertensive agents were also withdrawn on day one of the low calorie diet in order to avoid postural hypotension observed in Counterpoint and Counterbalance. The methodology can clearly be applied to other conditions which would be benefitted by substantial weight loss. The main ingredients of successful weight loss, apart from the use of a planned, short period of low calorie intake, were motivation on the part of the individual to achieve their goal and support from family and friends. The nutritional approach to avoiding weight 

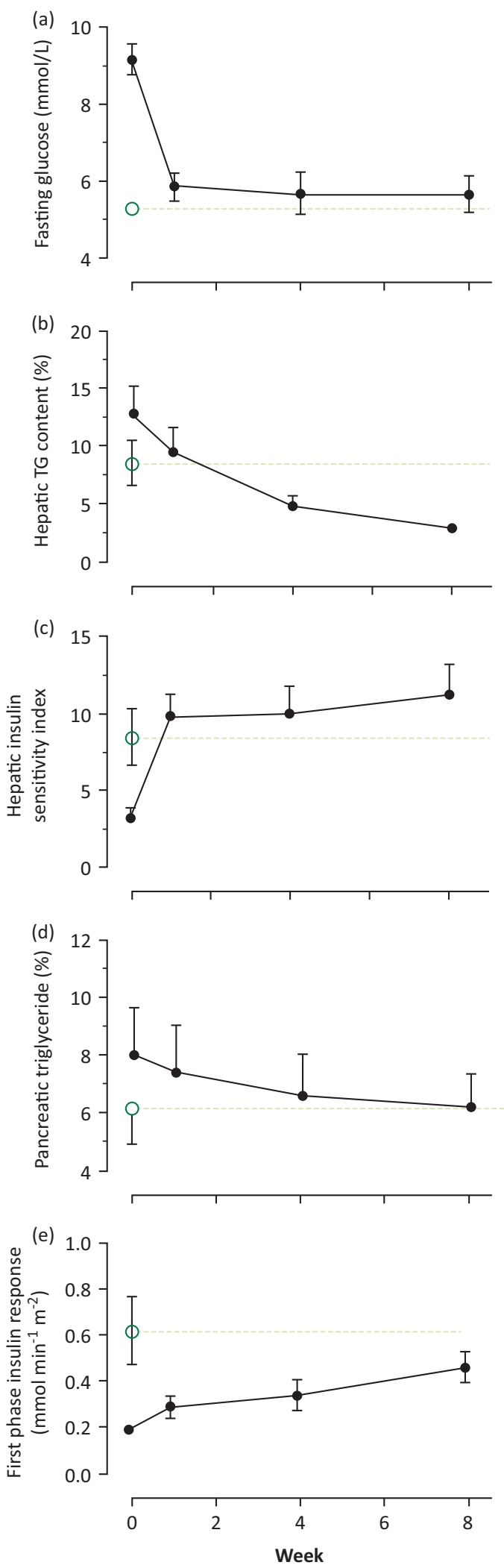

Fig 2. Data from Counterpoint showing (a) plasma glucose, (b) hepatic triglyceride content, (c) hepatic insulin sensitivity, (d) pancreas triglyceride content and (e) first phase insulin response. Data are shown as mean \pm standard error, for diabetic subjects (filled circles) and measured at a single time point for a weight matched non-diabetic control group. Figures reproduced with permission from Lim et al. ${ }^{9}$ regain has recently been summarised. ${ }^{15}$ Increase in exercise during the weight loss phase was discouraged as this tends to be accompanied by compensatory eating. This phenomenon explains why a portion of people fail to lose weight under conventional care. ${ }^{16}$ However, a steady increase in physical activity was strongly encouraged during weight maintenance. Recognition of this one-two approach for the very different phases of weight loss and weight maintenance is crucial.

\section{The pathophysiological basis of diabetes reversal}

The hypothesis-driven design of the Counterpoint study revealed surprisingly high liver fat in people with type 2 diabetes and a dramatic fall to normal on weight loss. This permitted a decrease in intra-pancreatic fat because of decreased export of very low density lipoprotein triglyceride from the liver and the return of normal first phase insulin response. It was possible to describe the new understanding of the aetiology of type 2 diabetes on the basis of Counterpoint, and the response of people with diabetes simply provided with information on how to reverse their condition. ${ }^{17}$ Counterbalance and DiRECT both extended these findings. Especially in DiRECT, the extent of the intra-organ fat accumulation in ordinary type 2 diabetes was underscored. In the intervention group, liver fat fell from $16.0 \% \pm 1.3 \%$ to $3.1 \%$ $\pm 0.5 \%$ and pancreas fat from $8.7 \% \pm 0.4 \%$ to $7.8 \% \pm 0.4 \%$ (Fig 3a). ${ }^{18}$ The importance of duration of type 2 diabetes since diagnosis was clear from the Counterbalance dataset, with $60 \%$ remission in those with duration $<4$ years, compared with $21 \%$ with duration $>8$ years. ${ }^{14}$ Even within the DiRECT study group, which only included people within the first 6 years after diagnosis of type 2 diabetes, those achieving non-diabetic levels of glucose control had a slightly but significantly shorter duration. ${ }^{18}$

The predictions of the twin cycle hypothesis have been confirmed, although the cellular basis for beta cell recovery could not be determined by the in vivo methodology employed. Independently, insight has been gained into the biology of return of insulin secretory capacity after decrease in exposure of beta cell to deleterious levels of triglyceride. Chronic in vitro exposure of beta cells to triglyceride or fatty acids decreases ability to respond to an acute increase in glucose levels ${ }^{19}$ and the basic concept that fat could impair beta cell function was far from new. ${ }^{20}$ Relatively low concentrations of saturated fatty acids can cause ultrastructural damage. ${ }^{21}$ This endoplasmic reticulum stress had been recognised in in vivo studies of type 2 diabetes. ${ }^{22,23}$ The added stress of raised plasma glucose is likely to exacerbate the metabolic insult once hyperglycaemia is established. ${ }^{24}$ Loss of specialised beta cell function due to dedifferentiation is now recognised as the most likely mechanism underlying type 2 diabetes. ${ }^{25-27}$ The old histology studies were certain to report apparently decreased numbers of beta cells as they relied upon insulin immunostaining, and the cells had stopped producing insulin although were still alive. Redifferentiation is permitted by removal of fat from within the pancreas coupled with lower delivery from plasma triglyceride. It is possible to tie in the histological and cell biological processes with observations during clinical reversal of the disease process. ${ }^{28}$ The new understanding of the mechanisms underlying type 2 diabetes fits well with several studies of significant weight loss in type 2 diabetes. ${ }^{29,30}$

It is important to recognise the internationally accepted threshold for diagnosis of diabetes $(\mathrm{HbA} 1 \mathrm{c}>48 \mathrm{mmol} / \mathrm{mol}$ or $6.5 \%$ ) and that a person has attained the state of post-diabetes 


\section{Roy Taylor}

Responders

Non-Responders

Controls

(a)
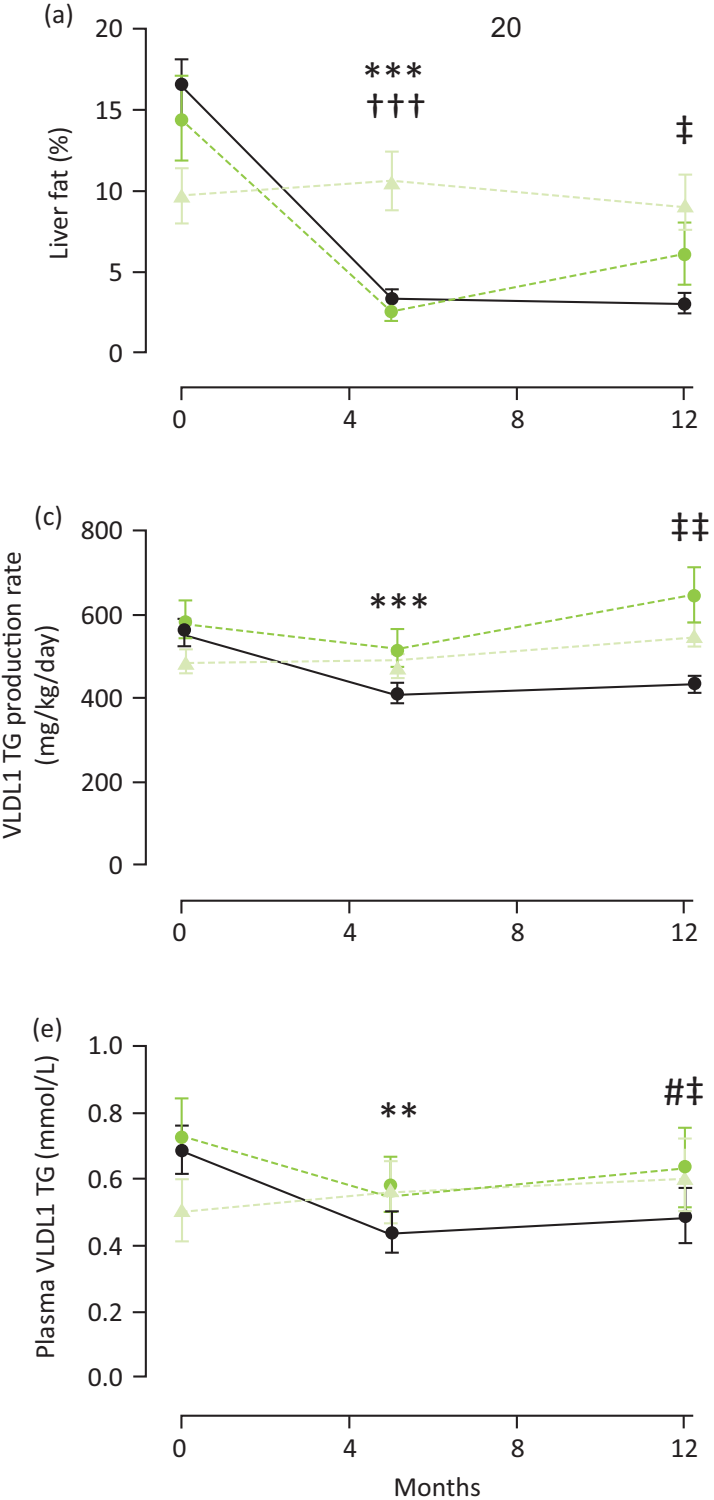

(b)

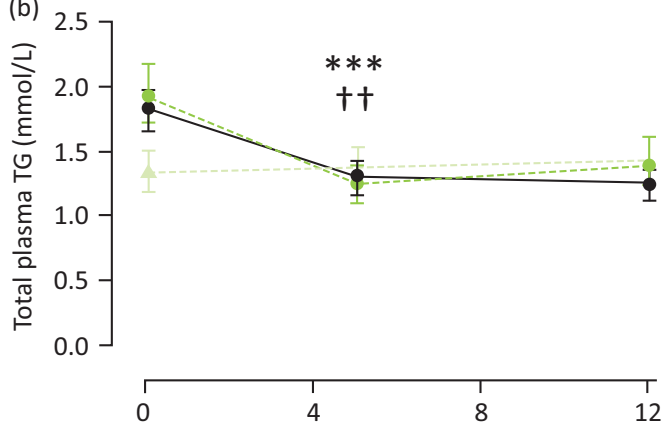

(d)

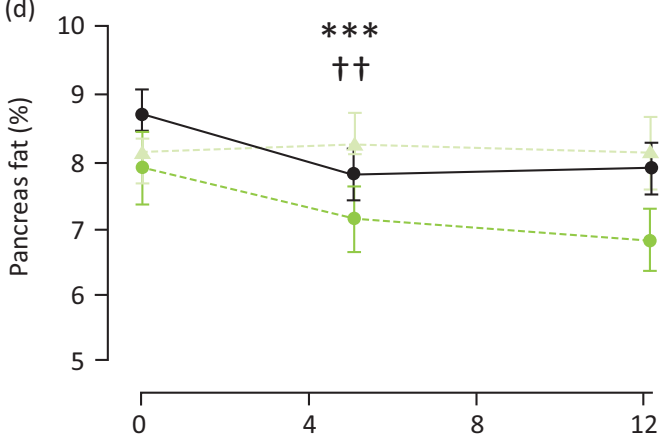

(f)

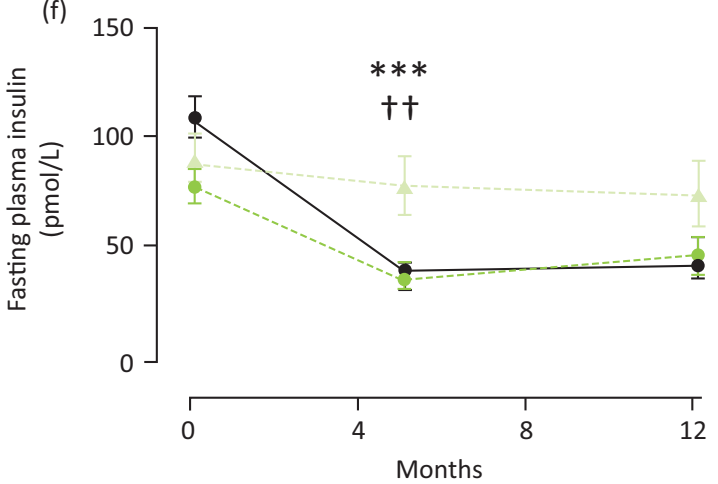

Fig 3. Data from DiRECT showing (a) hepatic triglyceride, (b) total plasma triglycerides, (c) hepatic VLDL1-TG production, (d) intrapancreatic triglyceride, (e) fasting plasma VLDL1-TG and (f) fasting plasma insulin. Time points are at baseline, post weight loss (5 months), and 12 months. Responders (those achieving non-diabetic glucose control; $n=40$ ) are shown as a solid line, and non-responders (those remaining in the diabetic range of $\mathrm{HbA1c}$ or plasma glucose) as a dotted mid green line. Those randomised to control (continuation of best practice by current guidelines) are shown as a dotted light green line. Figure reproduced with permission from Taylor et al. ${ }^{18}$

${ }^{* *} p<0.01$ vs. baseline, ${ }^{* * *} p<0.0001$ vs. baseline (responders)

${ }^{++} p<0.01$ vs. baseline, ${ }^{t++} p<0.0001$ vs. baseline (non-responders)

${ }^{\#} p<0.05$ vs. 5 months (responders); ${ }^{\ddagger} p<0.05$ vs. 5 months (non-responders); ${ }^{\neq} p<0.01$ vs. 5 months (non-responders)

if persistently below this level off all anti-diabetic agents. ${ }^{31}$ Post-diabetes is very different to pre-diabetes even though both may share similar glucose levels. The former is characterised by lower plasma lipids and lower blood pressure but the latter by high plasma lipids and blood pressure. In terms of 10 year vascular risk using QRISK, an average person with type 2 diabetes decreases from $23 \%$ to $7 \%$, with heart age for a group with average age 55 years decreasing from 71 to 56 years. ${ }^{32}$ Whereas pre-diabetes is unpredictably progressive, post-diabetes will remain constant long term providing weight remains steady 
and, predictably, reverts to type 2 diabetes if weight returns to the previous level.

\section{Further questions}

Publication of the Counterpoint study in 2011 elicited a major influx of enquiries from people with type 2 diabetes, and 'how to do it?' information was placed on a website. ${ }^{33}$ Subsequently, many individuals reported upon their own experience and 77 provided sufficient information to analyse. ${ }^{34}$ The majority were able to achieve normal glucose control having stopped their oral hypoglycaemic agents. Half of the group achieved this without medical help and in some cases against medical advice. Some individuals have established self-help networks and have placed their observations on the internet. One of the advantages of the information age is that doctors can listen directly to the comments of many people with a given condition.

Acceptance of the change of paradigm for type 2 diabetes by doctors and scientists was necessarily cautious in the early years after Counterpoint (2011), but especially following publication of DiRECT in 2017, the potential reversibility of type 2 diabetes became widely accepted. ${ }^{35}$ Recruitment for this study was rapidly over-subscribed, illustrating very well that health care professionals have underestimated the intense dislike of type 2 diabetes felt by sufferers. This became evident from the James Lind Alliance large scale survey of people with diabetes which showed their number one research priority to be 'Can type 2 diabetes be reversed and how can this best be achieved?'.32

A possible escape route from the condition is avidly accepted by many, to the surprise of their healthcare professionals who have been used to failure in conventional weight loss programmes. Reasons commonly cited for the keenness to try to regain health include removing the pejorative label of being 'diabetic', constant tablet taking and fear of complications. The principle difficulty for long term remission of type 2 diabetes is that of maintaining weight loss in the face of the slings and arrows of everyday life. The commonest pattern of weight regain in the first year of DiRECT was steady weight followed by rapid regain as a consequence of some major life event for an individual. The second year results of DiRECT will be available early in 2019, and this should provide further hard information about sustainability in this primary care population.

Several thousand emails have been received since publication of the Counterpoint study, and the most emotionally charged have come from people with type 2 diabetes but 'normal' body mass index (BMI) who were told by their doctor or nurse that it would be dangerous or unhealthy to lose weight. Some reported that they had lost weight despite the advice and had reversed their type 2 diabetes. ${ }^{34}$ The curious belief that individuals can be classified on the basis of an arbitrary cut off derived from population statistics is prevalent, even though a glance down any high street shows that people come in different size and shapes. For instance, it may be unwise to inform an elite rugby player that they are 'obese'. In the same way, an individual with a body type with a healthy BMI of, say, 20 may run into metabolic problems if it creeps up to $24 \mathrm{~kg} / \mathrm{m}^{2}$. $10 \%$ of people with type 2 diabetes happen to have a BMI less than $25 \mathrm{~kg} / \mathrm{m}^{2}$. In the nurses' health study there was a four-fold increase in prevalence of type 2 diabetes in women with a BMI of 23-25 compared with those who remained at less than $22 \mathrm{~kg} / \mathrm{m}^{2}{ }^{36}$ They had simply acquired more fat than they personally could cope with, and this has been described as exceeding their personal fat threshold. ${ }^{37}$ Beyond the level of the individual, some ethnic groups tend to have lesser capacity to store fat safely in the subcutaneous compartment, leading to excessive accumulation in the liver and then pancreas. The personal fat threshold concept is immediately understandable by patients as a way of explaining their condition and the way forward. As a hypothesis it requires experimental verification, and a study is in progress.

The question remains of why some individuals have beta cells susceptible to increased exposure to fatty acids. Many people have fatty liver disease and raised plasma triglyceride, but only a proportion of these go on to develop type 2 diabetes. Detailed genetic studies are required to define the basis of the evident degrees of susceptibility in different individuals. This should not be allowed to distract from the main point demonstrated in the studies discussed above: if a person has true type 2 diabetes, then they have more fat than their beta cells can tolerate.

\section{Summary}

Type 2 diabetes is a simple condition of having acquired more fat than the individual's body can cope with. Excess fat has accumulated in liver and pancreas and the individual's beta cells are susceptible to fat induced dedifferentiation. In a high proportion of those with short duration diabetes a robust and practical means has been developed to achieve or re-establish non-diabetic glucose control long term.

\section{Acknowledgements}

The research work of Drs Ee Lin Lim, Sarah Steven, Carl Peters, Ahmad Al-Mrabeh, Kieren Hollingsworth and Sviatlana Zhyzhneuskaya (all of the Magnetic Resonance Centre, Newcastle University) is gratefully acknowledged. DiRECT was carried out in partnership with Professor Michael Lean and the DiRECT team.

\section{References}

1 UKPDS Group. Effect of intensive blood-glucose control with metformin on complications in overweight patients with type 2 diabetes (UKPDS 34). Lancet 1998;352:854-65.

2 UKPDS Group. UK prospective diabetes study 16: overview of 6 years' therapy of type II diabetes: a progressive disease. Diabetes 1995:44:1249-58.

3 Rudenski AS, Hadden DR, Atkinson AB et al. Natural history of pancreatic islet B-cell function in type 2 diabetes mellitus studied over 6 years by homeostasis model assessment. Diabet Med 1988;5:36-41.

4 Rahier ], Guiot Y, Goebbels RM, Sempoux C, Henquin JC. Pancreatic beta cell mass in European subjects with type 2 diabetes. Diabetes Obes Metab 2008;10(Suppl 4):32-42.

5 Petersen KF, Dufour S, Savage DB et al. The role of skeletal muscle insulin resistance in the pathogenesis of the metabolic syndrome. Proc Natl Acad Sci U S A 2007;104:12587-94.

6 Petersen KF, Dufour S, Befroy D et al. Reversal of non-alcoholic hepatic steatosis, hepatic insulin resistance, and hyperglycemia by moderate weight reduction in patients with type 2 diabetes. Diabetes 2005;54:603-8.

7 Ravikumar B, Gerrard J, Dalla Man C et al. Pioglitazone decreases fasting and postprandial endogenous glucose production in proportion to decrease in hepatic triglyceride content. Diabetes 2008:57:2288-95

8 Taylor R. Pathogenesis of type 2 diabetes: Tracing the reverse route from cure to cause. Diabetologia 2008;51:1781-9. 
9 Lim EL, Hollingsworth KG, Aribisala BS et al. Reversal of type 2 diabetes: normalisation of beta cell function in association with decreased pancreas and liver triacylglycerol. Diabetologia 2011;54:2506-14.

10 Steven S, Hollingsworth KG, Al-Mrabeh A et al. Very low calorie diet and 6 months of weight stability in type 2 diabetes: Pathophysiological changes in responders and nonresponders. Diabetes Care 2016;39:158-65.

11 Lean ME, Leslie WS, Barnes AC et al. Primary care-led weight management for remission of type 2 diabetes (DiRECT): an open-label, cluster-randomised trial. Lancet 2017;391:541-51.

12 Sjostrom L, Peltonen M, Jacobson P et al. Association of bariatric surgery with long-term remission of type 2 diabetes and with microvascular and macrovascular complications. JAMA 2014;311:2297-304.

13 Lingvay I, Guth E, Islam A, Livingston E. Rapid improvement in diabetes after gastric bypass surgery: is it the diet or surgery? Diabetes Care 2013;36:2741-7

14 Steven S, Hollingsworth KG, Small P et al. Weight loss decreases excess pancreatic triacylglycerol specifically in type 2 diabetes. Diabetes Care 2016:39:158-65.

15 Taylor R, Barnes A. From new understanding of type 2 diabetes to practical management. Diabetologia 2018;61:273-83.

16 Finlayson G, Bryant E, Blundell JE, King NA. Acute compensatory eating following exercise is associated with implicit hedonic wanting for food. Physiol Behav 2009;97:62-67.

17 Taylor R. Type 2 diabetes: etiology and reversibility. Diabetes Care 2013;36:1047-55

18 Taylor R, Al-Mrabeh A, Zhyzhneuskaya S et al. Remission of human type 2 diabetes requires decrease in liver and pancreas fat content but is dependent upon capacity for beta cell recovery. Cell Metab 2018;28:1-10.

19 Lee $\mathrm{Y}$, Hirose $\mathrm{H}$, Ohneda $\mathrm{M}$ et al. B-cell lipotoxicity in the pathogenesis of non-insulin-dependent diabetes mellitus of obese rats: impairment in adipocyte-B-Cell relationships. Proc Natl Acad Sci U S A 1994;91:10878-82.

20 Unger RH. Lipotoxicity in the pathogenesis of obesity-dependent NIDDM. Genetic and clinical implications. Diabetes 1995;44:86370.

21 Pinnick K, Neville M, Clark A, Fielding B. Reversibility of metabolic and morphological changes associated with chronic exposure of pancreatic islet beta cells to fatty acids. J Cell Biochem 2010;109:683-92.

22 Huang C], Lin CY, Haataja L et al. High expression rates of human islet amyloid polypeptide induce endoplasmic reticulum stress mediated beta-cell apoptosis, a characteristic of humans with type 2 but not type 1 diabetes. Diabetes 2007;56:2016-27.
23 Laybutt DR, Preston AM, Akerfeldt MC et al. Endoplasmic reticulum stress contributes to beta cell apoptosis in type 2 diabetes. Diabetologia 2007;50:752-63.

24 Poitout V, Amyot ], Semache M et al. Glucolipotoxicity of the pancreatic beta cell. Biochim Biophys Acta Mol Cell Biol Lipids 2010:1801:289-98

25 Brereton MF, Iberl M, Shimomura K et al. Reversible changes in pancreatic islet structure and function produced by elevated blood glucose. Nat Commun 2014;5:4639.

26 Talchai C, Xuan S, Lin HV, Sussel L, Accili D. Pancreatic beta cell dedifferentiation as a mechanism of diabetic beta cell failure. Cell 2012;150:1223-34.

27 White MG, Marshall HL, Rigby R et al. Expression of mesenchymal and $\alpha$-cell phenotypic markers in islet $\beta$-cells in recently diagnosed diabetes. Diabetes Care 2013:36:3818-20.

28 White MG, Shaw JAM, Taylor R. Type 2 diabetes: the pathologic basis of reversible beta-cell dysfunction. Diabetes Care 2016;39:2080-8.

29 Henry RR, Wallace P, Olefsky JM. Effects of weight loss on mechanisms of hyperglycaemia in obese non-insulin dependent diabetes mellitus. Diabetes 1986;35:990-8.

30 Wing RR, Koeske R, Epstein LH et al. Long-term effects of modest weight loss in type II diabetic patients. Arch Intern Med 1987:147:1749-53.

31 McCombie L, Leslie W, Taylor $\mathrm{R}$ et al. Beating type 2 diabetes into remission. BMJ 2017;358:j4030.

32 Taylor R, Barnes AC. Can type 2 diabetes be reversed and how can this best be achieved? James Lind Alliance research priority number one. Diabet Med 2018; In press.

33 Newcastle Magnetic Resonance Centre. Reversing type 2 diabetes. Newcastle: Newcastle Magnetic Resonance Centre, 2011. http:// go.ncl.ac.uk/diabetes-reversal. [Accessed 03 September 2018].

34 Steven S, Lim E, Taylor R. Population response to information on reversibility of type 2 diabetes. Diabet Med 2013;30:e135-8.

35 Leslie WS, Ford I, Sattar N et al. The Diabetes Remission Clinical Trial (DiRECT): protocol for a cluster randomised trial. BMC Fam Pract 2016;17:20.

36 Hu FB, Manson JE, Stampfer MJ et al. Diet, lifestyle, and the risk of type 2 diabetes mellitus in women. N Engl J Med 2001;345:790-7.

37 Taylor R, Holman R. Normal weight individuals who develop type 2 diabetes: the personal fat threshold. Clin Sci 2015;128:405-10.

Address for correspondence: Professor Roy Taylor, Newcastle University, Magnetic Resonance Centre, Campus for Ageing and Vitality, Newcastle upon Tyne, NE4 5PL, UK.

Email: roy.taylor@ncl.ac.uk 